\section{CONTRACTILE PROTEINS}

\section{Muscular Platelets}

from our Cell Biology Correspondent

Now that an actin-like protein, which may well prove to be completely identical to muscle actin, has been found in considerable amounts in nerve cells (Nature New Biology, 234, 115; 1971) where it may be responsible for the motility of the growing tips of axons, it comes as no surprise to read in the current issue of the Proceedings of the US National Academy of Sciences (68, 2703 ; 1971) that Adelstein, Pollard, and Kuehl have isolated a myosin-like as well as an actin-like protein from human blood platelets.

Platelets play a crucial part in wound healing; they cause the retraction of clots and clearly such a contractile function must depend on some sort of molecular contractile apparatus. It is several years now since so-called thrombosthenin-a complex mixture of contractile proteins-was isolated from platelets but successive attempts to isolate actin or myosin-like proteins from this material have resulted in failure. Adelstein and his colleagues have, however, now succeeded in this by dissolving thrombosthenin in a buffer containing $\mathrm{Mg}^{2+}$, ATP and dithiothreitol and fractionating it by ammonium sulphate precipitation followed by chromatography and polyacrylamide gel electrophoresis.

By these procedures they have isolated proteins which can be classified as myosin-like by several criteria. The myosin-like protein from platelets, for instance, binds both muscle and platelet actin; it has an $\mathrm{Mg}^{2+}$ dependent ATPase activity and in the electron microscope it can be seen to have the characteristic structure of myosin with a rod and a head portion, the head portion binding actin. Furthermore, the major polypeptide chain of platelet myosin has a molecular weight of about 200,000 daltons, the same as that of rabbit skeletal muscle myosin. As Adelstein et al. comment, the predominance of head and rod portions of myosin molecules in their preparations suggests that platelet myosin is probably subject to proteolytic cleavage either exclusively during its isolation or in vivo before isolation. The suggestion that thrombin may be responsible for the cleavage of thrombosthenin proteins in platelets has, of course, been made repeatedly in the past.

Apart from intact myosin and myosin head and rod pieces, the fourth chief protein which Adelstein et al. have isolated from thrombosthenin assembles into filaments $6 \mathrm{~nm}$ wide which form "arrowhead" complexes with platelet and skeletal myosin. This protein has the same molecular weight as skeletal muscle actin, 46,000 daltons, and it contains one 3-methylhistidine residue per molecule. For these reasons Adelstein and his colleagues believe it is an actin.

Clearly, fingerprint analyses of these two platelet contractile proteins and, ultimately, analyses of their amino-acid sequences will have to be made in order to establish their complete identity or otherwise with their skeletal muscle counterparts. But from the evidence currently available it is reasonable to conclude that the molecular basis of contraction in platelets is in all essentials similar to that of skeletal muscle.

\section{Martera bctince \\ Metal Matrix Composites}

from a Correspondent

THE objective of a symposium on November 15 at the Liverpool Polytechnic on the subject of metal matrix composite materials was to bring together research workers and potential consumers for an exchange of current views on the usefulness of these composite systems and possible future developments. It was clear that although experimental work (such as that described by Dr B. Harris of the University of Sussex) is still being carried out on metals reinforced with strong metal wires, present interest is largely in systems in which carbon fibres or refractory whiskers or fibres are the reinforcing agents. The obvious reason for this is the potential saving in weight to be gained from using these light fibres instead of the denser metallic reinforcements.

The dominant theme of the symposium was undoubtedly the problem of the high temperature instability of carbon fibres and whiskers in contact with matrix metals such as nickel and aluminium. Experiments with carbon fibres have so far seemed to concentrate on the use of nickel or aluminium as the matrix metal. Dr S. J. Harris (University of Nottingham) explained that on the basis of cost, density and general properties, the aluminium/carbon fibre system is probably the most promising for study, and he described the methods used at Nottingham and Rolls Royce for producing good composites. $\mathrm{He}$ discussed the properties of these materials, in comparison with such competitors as boron fibre-reinforced aluminium and whisker-reinforced aluminium, but was forced to conclude that experimental strengths are often lower than theoretically obtainable values, usually because of fibre damage during manufacture.

Work on carbon fibres in nickel described by Dr W. Bonfield (Queen Mary College, London) and $\mathrm{Mr} \mathrm{P}$. Ormiston (University of Southampton) left listeners with the impression that the lack of high temperature compatibility in this system, which leads to fibre degradation especially in the presence of oxygen, probably precludes its commercial use unless some means could be found of introducing an efficient diffusion barrier between fibre and matrix. There was considerable discussion of the

\title{
Specificity of mRNA Degradation
}

How messenger RNAs are degraded in bacteria and how the degradation process is regulated remain vexed questions. There is, of course, no shortage of hypotheses and there are one or two enzymatic activities which are likely candidates for the job of cutting messenger RNAs to pieces, but the biochemistry of the process remains to be fully elucidated and its elucidation is clearly not going to be any easy matter. As Marrs and Yanofsky report in next Wednesday's Nature New Biology, different messengers in the same Escherichia coli cell are degraded at different rates and this observation leads them to suggest that the rate of degradation of any messenger may be partly determined by its particular structure.

Marrs and Yanofsky have measured the half-lives of phage T7 mRNAs and tryptophan operon messengers in $E$. coli cells infected with an amber mutant of T7, am193. This phage, because of the amber mutation it carries, does not make its own T7 RNA polymerase and as a result the phage late genes are not expressed. All RNA synthesis in the cells infected with am193 is carried out by the $E$. coli DNA dependent RNA synthetase and so it is sensitive to inhibition by rifampicin.

Using this experimental system Marrs and Yanofsky have compared the synthesis and survival of the early T7 messengers and the $E$. coli tryptophan operon messengers in the same cell. In short, they find that whereas the T7 messenger RNAs have half-lives of some 6 to 20 minutes, the trypE messenger RNA has a half-life of a mere 80 seconds in the infected cells as it does in normal uninfected $E$. coli.

Marrs and Yanofsky's observation clearly indicates that the enzymatic machinery responsible for degrading messenger RNAs attacks different classes of messengers at different rates; in other words, the degradation machinery must be able to distinguish one messenger from another and any hypothesis seeking to account for the turnover of messengers must be able plausibly to account for this fact. 